\title{
Investigación educativa en procesos de enseñanza y aprendizaje*
}

\author{
Educational research in teaching and learning processes \\ Pesquisa educativa em processos de ensino e aprendizagem
}

DOI: http://dx.doi.org/10.21803\%2Fpenamer.11.21.531

Ciro E. Redondo Mendoza

https://orcid.org/0000-0002-2357-7289

\section{¿Cómo citar este artículo?}

Redondo, C. (2018). Investigación educativa en procesos de enseñanza y aprendizaje. Pensamiento Americano, 11(21), 153-168

DOI: http://dx.doi.org/10.21803\%2Fpenamer.11.21.531

\section{Resumen}

El presenteartículo de investigación se desarrolló con el objetivo deconocer la innovación de enseñanzas y aprendizajes en las prácticas investigativas de docentes de la Ciudad de Medellín; Metodología. Desde el análisis de una investigación cualitativa, el desarrollo del estudio se alineo con el método hermenéutico interpretativo, fundamentado en el aprendizaje significativo, así como en los procesos de enseñanza y aprendizaje en prácticas investigativas de docentes. La información se obtuvo mediante las observaciones directas y entrevistas semiestructuradas a cuatro (4) docentes, los cuales fueron escogidos por su antigüedad dentro de las instituciones a las cuales pertenecen. La técnica de análisis empleada fue la triangulación de los datos. Resultados generales. La innovación implica un cambio intencional y se refiriere a un proceso orientado hacia la mejora, por ello, es fundamental para el estudio de la investigación escolar como una estrategia pedagógica que representa el último enfoque de investigación en el aula. Entre las reflexiones finales puede decirse que la innovación en instituciones educativas, tiene una íntima relación con el sector tecnológico; es el resultado, además, de acciones preestablecidas; que orientan hacia una adaptación flexible, hacia la experimentación, y el cambio guiado y por ello, los docentes han de estar en capacidad de alentar la investigación, la participación y la innovación en el alumnado.

PALABRAS CLAVE: Innovación - Procesos de enseñanza y aprendizaje - Prácticas investigativas.

\begin{abstract}
The present research article was developed with the objective to know the innovation of teachings and learning in the investigative practices of teachers in the City of Medellin; Methodology. From the analysis of a qualitative research, the development of the study was aligned with the interpretive hermeneutic method, based on meaningful learning, as well as on teaching and learning processes in teacher research practices. The information was obtained through direct observations and semistructured interviews with four (4) teachers, who were chosen for their seniority within the institutions which they have belonged to. The analysis technique used was triangulation. General results. Innovation implies an intentional change and refers to a process oriented towards improvement, therefore, it is fundamental for the study of school research as a pedagogical strategy that represents the last research approach in the classroom. Among the final reflections it can be said that innovation in educational institutions has an intimate relationship with the technological sector; it is the result, in addition, of preestablished actions; that guide towards a flexible adaptation, towards experimentation, and guided change and, therefore, teachers must be able to encourage research, participation and innovation in the students.

KEYWORDS: Innovation - Teaching and learning processes - Research practices.
\end{abstract}

* El presente artículo se deriva del trabajo presentado como requisito final para optar al título de Doctor en Ciencias de la Educación, titulado "innovación en los procesos de enseñanza y aprendizaje en prácticas investigativas de docentes de la ciudad de Medellín" 


\section{Resumo}

Introdução. O presente artigo de pesquisa se desenvolveu com o objetivo de conhecer a inovação de ensinos e aprendizagens nas práticas de pesquisa de docentes da Cidade de Medelín. Metodologia. Desde a análise de uma pesquisa qualitativa, o desenvolvimento do estudo se alinhou com o método hermenêutico interpretativo, fundamentado na aprendizagem significativa, assim como os processos de ensino e aprendizagem em práticas de pesquisa de docentes. A informação foi obtida diante de observações diretas e entrevistas semiestruturadas a quatro docentes, os quais foram escolhidos por sua longevidade dentro das instituições as quais pertencem. A técnica de análise utilizada foi a triangulação dos dados. Resultados gerais. A inovação implica uma mudança intencional e se refere a um processo orientado para a melhoria, por isso, é fundamental para o estudo a pesquisa escolar como uma estratégia pedagógica que representa o último enfoque de pesquisa em aula. Entre as reflexões finais se pode dizer que a inovação em instituições educativas tem uma íntima relação com o setor tecnológico; é o resultado, ademais, de ações pré-estabelecidas; que orientam a uma adaptação flexível para um experimento, e para a mudança gerada por ele. Os docentes têm que estar de posse da capacidade de instigar a pesquisa, a participação e a inovação nos alunos.

PALAVRAS-CHAVE: Inovação - processos de ensino e aprendizagem práticas de pesquisa.

\section{Perfil}

Doctor en ciencias de la educación de la universidad Rafael Belloso Chacín, magister en Educación con mención en Psicología Educativa de la Universidad Peruana Unión, , profesor de corporación Universitaria Minuto de Dios e investigador de la Corporación Universitaria Adventista. ciro0980@hotmail.com

\section{Ciro E. Redondo Mendoza}

Licenciado el teología y psicología. 


\section{Introducción}

La innovación en los escenarios educativos permite movilizar y encauzar esfuerzos para lograr trasformaciones importantes, garantizando así la permanencia de las instituciones en el tiempo, convirtiéndolas en líderes en el contexto al que pertenecen además de un referente para sus colaboradores (Rodríguez, 2010). Ello implica planificar y enseñar bajo un enfoque estratégico transmitiendo detalles que inspiren procesos de aprendizaje innovadores, los cuales emergen de los actores educativos, cuyas inquietudes deben ser orientadas a sus deseos basados en las experiencias, proyecciones de cambio, cambios sociales, otros, para desencadenar un desarrollo de forma continua y progresiva.

Es así como, la innovación presume la acción de profesionales de la educación con suficientes conocimientos e información, así como la experiencia para conducir con éxito a sus estudiantes, quienes asumen tanto las acciones como las reacciones del líder como modelo a seguir. Por ello, como lo menciona de la Fuente, Martínez, y Cardelle-Elawar, (2018). la innovación necesita personas con imaginación, diligentes, con noción de la organización, de su estructura, normas y contenidos programáticos, siendo capaz de involucrar a todos en el proceso de enseñanza aprendizaje.

En consecuencia, las instituciones educativas requieren de profesionales con capacidad de liderazgo para generar innovaciones tomando en cuenta la combinación de habilidades, roles y tareas que conlleva educar, estos además deben ajustarse a las fases evolutivas de la investigación lo cual incluye: la diagnosis de una situación, la teorización y análisis, la fase metodológica, recolección y análisis de información, hallazgos, consideraciones del autor, recomendaciones, aportes, teorías sustantivas, otras fases que el investigador conven- ga necesario. (Rodríguez, G., Gil, J., \& García, E.,1999).

De manera que, innovar en los procesos de enseñanza - aprendizaje en las practicas investigativas de docentes específicamente en la ciudad de Medellín, adaptando las destrezas que se transmiten en la educación a los nuevos y dinámicos requerimientos laborales, educar para la ciudadanía y contribuir a la competitividad son algunos de los retos que el sistema educativo enfrenta desde la nueva sociedad del conocimiento (Mora, 2004). Es allí donde la presente investigación está interesada en conocer las prácticas innovadoras que lleven a transformar los procesos de enseñanza y aprendizaje en las organizaciones educativas en las prácticas investigativas.

La actual investigación pretende dar respuesta a los planteamientos que el investigador hace con respecto conjeturas que emergen de las experiencias vividas, observaciones participativas no sistematizadas y del proceso de revisión bibliográfica, acciones que permitieron direccionarla hacia el logro de los alances formulados en la misma (Cordero, 2013).

De allí, el autor se plantea los siguientes interrogantes: ¿Qué entiende por innovación de los procesos de enseñanza - aprendizaje?, ¿Cuáles son las dimensiones que impulsan la innovación de los procesos de enseñanza aprendizaje en las prácticas investigativas de los docentes?, ¿Qué tipos de cambios impulsados por la innovación participan en las prácticas investigativas de los docentes?,

¿Cuáles son las características del proceso de cambio para producir innovación en el proceso de enseñanza aprendizaje?, ¿Qué factores influyen en el fracaso y en el éxito de la innovación de los procesos de enseñanza aprendizaje en las prácticas investigativas de los docentes?, ¿Cómo desarrollan las prácticas in- 
vestigativas los docentes para llegar a procesos de enseñanza aprendizaje innovadores?

\section{Diseño de la investigación}

El enfoque hermenéutico, dada su naturaleza cualitativa, necesita un diseño general congruente con el arte de interpretar, en la medida que se vaya identificando el escenario objeto que se pretende estudiar en la interacción con los individuos con los cuales cohabitan en el contexto, y guie el estudio hacia el logro de sus fines para responder el problema planteado; Bonilla, Castro y Rodríguez (2005, p. 127). En ese sentido, auxiliado en los modelos de Bonilla y Rodríguez, en sus primeras etapas con las teorías descrita por Martínez (2010), para las etapas de análisis de los datos y de teorización, se ha adaptado el presente diseño para realizar el proceso de investigación. Se basa en una estructura circular la cual abarca seis momentos, iniciando en la Fase Preparatoria donde se examina la situación objeto de estudio, se detalla el escenario de los acontecimientos, culminando con el momento que generaliza las teorías.

Por su parte, su estructura inicial, es circular, integral, operativa hasta la fase de las acciones investigativas; es un proceso de múltiples entradas que se retroalimenta de la práctica y el conocimiento, esta práctica va obteniendo del contacto con el escenario junto a los individuos que interactúan en torno a ella. El camino hermenéutico que abarca todo el proceso será el siguiente:

Momento I: Fase Preparatoria: crea un camino hacia una aproximación reflexiva a la investigación; indagación de la situación, delineación del escenario; incógnitas de la investigación, propósitos y justificación. Momento II: Fase de Diseño: donde se explica el método aplicado en esta investigación; el Enfoque Epistemológicoy el Diseño de la Investigación. Momento III: Teorías de Entrada: Comprende el Marco Teórico Referencial. Momento IV: Trabajo de campo: las técnicas de recolección de los datos que se aplicaran; la ordenación de los datos, la certificación, la confiabilidad de los mismos.

Momento V: Hallazgos y Finales: se realiza el análisis de los datos para avanzar a la etapa de teorización; Generalización de la Teoría: Se busca que el diseño de la presente investigación acoja un proceso dialéctico, explicativo, holista circular, en forma secuencial y pormenorizada, establecer los momentos los cuales cubre el camino a seguir para conocer la innovación de los procesos de enseñanza y aprendizaje en práctica investigativa de docentes de la ciudad de Medellín (Higuera, 2013).

\section{Enfoque epistemológico}

Todo estudio investigativo envuelve la necesidad de situarla en un marco epistemológico, permite en lo sucesivo posibilitar la ruta metodológica la cual guiará el proceso del quehacer investigativo; de forma que, la presente investigación se ubicará en el Enfoque Cualitativo, cuya naturaleza permite elaborar un proceso dialéctico, constructivista sistémico, (Arnold, 1997). a través de los métodos intentando dar respuesta a los propósitos de la misma; es decir, para conocer la innovación de los proceso de enseñanza y aprendizaje en práctica investigativa de docentes de la ciudad de Medellín.

De allí, la teoría que se origine; es decir una teoría sustantiva real, original, autónoma, sin ataduras con el poder político, será un producto social influenciada por los valores, basada en el entendimiento de la realidad que viven como seres humanos, esto como consecuencia de un proceso histórico el cual redime la diversidad tanto como la particularidad, esta influye en sus comportamientos, lo cual viene de lo que ellos son como poder cognitivo y miembros de una organización mayor llamada nación colombiana. 
En consecuencia, lo analizado se cristianizará en nuevo conocimiento o teoría, esta se creará desde la realidad y sus protagonistas, llegando al ser cognoscente desde su propia episteme, cargada de subjetividad e intersubjetividad. En efecto, estos presupuestos, de carácter ontológico se explicitarán dentro de una episteme absolutamente necesaria, donde desde la metodología además de las técnicas que se aplicaran, como igualmente, a las reglas de interpretación comprenden el método hermenéutico.

\section{El método}

El método escogido para efectuar esta investigación será el método hermenéutico interpretativo, (Arráez, Calles, y Moreno, 2006) el cual acercará a la comprensión de la realidad a través de un ciclo dialéctico la cual va de la data a la interpretación y de ésta a los datos para darle significado intersubjetivamente a los mismos.

Es pertinente esclarecer, con la intención de iniciar el estudio, se comenzara utilizando el modelo positivista, pero al progresar en el campo epistemológico, se espera descubrir de qué forma las teorías sustantivas las cuales fundamentan el constructo a desarrollar deban ser interpretadas hermenéuticamente, para llegar a trasladar al campo educativo los fundamentos del Aprendizaje Significativo del Constructivismo, a la nueva teoría que emerja del proceso de análisis e interpretación de los datos recolectado.

En consecuencia, el método hermenéutico, a utilizar en este estudio se alineará a observar la realidad; sus fenómenos y actores; compilarlos datos e información de manera sistemática, descifrarlos dialécticamente, así como analizarlos para, a través de la codificación y categorización de dichos datos; así como de su estructuración, además de la teorización surge el constructo Innovación en el proceso de enseñanza aprendizaje.
En ese sentido, Strauss y Corbin (2002, p. 24) explican que para teorizar no sólo hay que admitir e intuir ideas o conceptos; sino también crearlos en un esquema racional, metódico y interpretativo. En el fondo de la teorización subyace la interacción entre hacer inducciones: emanar conceptos, sus dimensiones, principios a partir de la interpretación de los datos y de procesos de observación; "al final el investigador, desarrolla una teoría sistemáticamente, convirtiendo los productos del análisis en una teoría".

En consecuencia, el método aplicado en esta investigación, se concentrará en la categorización lógica de las interpretaciones del significado que se originen de las interacciones con los sujetos en un contexto ya escogido para tal fin; las instituciones educativas seleccionadas y su contexto.

Es decir, la investigación residirá en analizar las teorías las cuales puedan originar el constructo Innovación educativa, para descifrar sus significados, entendiendo de la mejor manera los comportamientos, los escritos, contenidos, vocabularios, lecciones, las cuales se observarán en el escenario, para llegar a la argumentación científica de la teoría emergente, desde una lógica interactiva, conservando la singularidad del contexto del cual forma parte.

\section{Muestreo}

La muestra estará conformada por una suficiente cantidad de individuos, hipótesis, sucesos, documentos y demás elementos presentes en el contexto que se manifiesten o se relacionen con los procesos de innovación de los procesos de enseñanza y aprendizaje en las practicas investigativa de los docentes de la ciudad de Medellín, esto con el fin de optimizar los procesos en busca de mejorar su calidad y por ende mejorar sus resultados.

De esa forma, esta investigación siguiendo las 
señales de los códigos y categorías emergentes (muestreo teórico), para la selección de los entrevistados o fuentes de información se utilizará, el patrón que indique la necesidad de explorar las categorías al detalle, concluyendo este camino cuando cada categoría haya sido explorada rigurosamente, elaborándose e integrándose en la nueva teoría. (Krause, 1995).

\section{Recolección de datos}

La recolección de los datos estuvo dirigida a cómo los informantes interpretan, analizan y comprenden la realidad dentro de los procesos del contexto donde normalmente desarrollan sus acciones, en este particular, las Instituciones Educativas de la Ciudad de Medellín, seleccionadas para el estudio, conducente a que los resultados pasan a ser la razón principal, que impulsa a conocer las implicaciones en materia de Innovación de los Procesos de enseñanza y aprendizaje en prácticas investigativas de docentes. Es el momento donde participantes del proceso educativo, expresan su punto de vista, basado en sus conocimientos y vivencias, con respecto al tema abordado.

En definitiva, tanto la observación como la entrevista permitió la pertinencia o no de conocer la innovación de enseñanzas y aprendizajes en las prácticas investigativas de docentes de la Ciudad de Medellín; así como la manera en que los participantes de la investigación estuvieron comprometidos con las acciones involucradas, permite medir el nivel de compromiso de cada uno, así como su grado de satisfacción, factores inciden en los resultados obtenidos.

\section{Técnicas de recolección de datos}

Se empleó un instrumento para la recolección de los datos, seleccionado en relación al método de investigación; de tal manera, que la técnica aplicada en este estudio fue una entrevista semiestructurada, que permitió suministrar la data necesaria para el análisis, de acuerdo a esto, la estrategia permite conocer el fenómeno, tomando en cuenta sus acontecimientos, los cuales provienen de la experiencia y conocimientos de los entrevistados.

Desde esta perspectiva, el análisis e interpretación de la información demandó de la implementación de técnicas de estudio, más tradicionales entre ellas, la toma de nota rápida y las grabaciones de audio, las cuales en criterio de Martínez (1999), son una manera de reconocer estructuralmente la teoría que se derivada de distintas fuentes, procedimientos, observadores, actores, textos, otros; lo que facilita al investigador el poder observar y analizar los hechos cuantas veces lo requiera, con la posibilidad de recurrir a otros investigadores si se necesitara ayuda.

Posteriormente, estas notas son revisadas y analizadas, tomando los aspectos más relevantes de cada entrevista, sin alterar las ideas manifestadas por los informantes. En este sentido, una vez que se decidió que tipo de entrevista y cuáles fueron los sujetos clave, se llevaron a cabo, a saber, con un total de cinco informantes, cuyos detalles se desarrollan a continuación.

\section{Hallazgo, reflexiones finales y recomenda- ciones}

Como propósito general el investigador se planteó Conocer la innovación de enseñanzas y aprendizajes en las prácticas investigativas de docentes de la Ciudad de Medellín, cuya población de estudio estuvo conformada por docentes que protagonizaron prácticas investigativas en las diferentes instituciones educativas de la ciudad, finalmente se dio respuesta a las interrogantes consideradas al inicio de la investigación, por medio del proceso de triangulación, donde se señalaba ¿Qué entiende por innovación de los procesos de enseñanza aprendizaje?, así como otras con las que se pretendió alcanzar el propósitos general. 
Apoyado en las experiencias y conocimientos de los informantes, se formularon otras interrogantes de interés como ¿Cuáles son las dimensiones que impulsan la innovación de los procesos de enseñanza aprendizaje en las prácticas investigativas de los docentes?, ¿Qué tipos de cambios impulsados por la innovación participan en las prácticas investigativas de los docentes?,

¿Cuáles son las características del proceso de cambio para producir innovación en el proceso de enseñanza aprendizaje?, ¿Qué factores influyen en el fracaso y en el éxito de la innovación de los procesos de enseñanza aprendizaje en las prácticas investigativas de los docentes?, ¿Cómo desarrollan las prácticas investigativas los docentes para llegar a procesos de enseñanza aprendizaje innovadores?

Ahora bien, una vez que se contestaron las interrogantes, se presentan los hallazgos que se originan del proceso de investigación, ocasionando el análisis de la información a partir del primer momento que se mantuvo en vinculación con los participantes, pasando por todo un proceso teórico y de estudio, los cuales son expuestos a continuación:

Hallazgo 1. El liderazgo que ejercen los directivos docentes en cada una de esas instituciones, se maneja de manera tradicional en cuanto a la disciplina, la organización, la administración, resaltando que existe un sentido de pertenencia, con interés en las investigaciones en el ámbito nacional e internacional, las cuales permiten mucha reflexión sobre el acto educativo.

Hallazgo 2. La innovación implica un cambio intencional, ya que un cambio a secas, puede darse más allá de la voluntad de los agentes. Al mismo tiempo el cambio que origina una innovación reviste un carácter positivo, de mejor, es decir, no se realiza una innovación con el fin de empeorar una situación.

Hallazgo 3. Innovación se refiriere a un proceso orientado hacia la mejora, cabe preguntar para quiénes es una mejora, por lo que es necesario entender quién se ha beneficiado de una determinada mejora, así como definir de manera precisa en qué consiste esa mejora.

Hallazgo 4. Se debe considerar la posibilidad de usar materiales nuevos o actualizados (recursos relacionados directamente con la enseñanza, como materiales curriculares o tecnologías); además de la factibilidad de utilizar nuevos abordajes de enseñanza (nuevas estrategias o actividades de enseñanza); y tercero la posibilidad de alterar las creencias (concepciones y teorías pedagógicas que subyacen en algunos nuevos programas o políticas).

Hallazgo 5. La innovación es como una serie de intervenciones, decisiones y procesos, con cierto grado de intencionalidad y sistematización, que tratan de modificar actitudes, culturas, contenidos, ideas, modelos y prácticas pedagógicas, a su vez, de introducir en una línea renovadora, nuevos proyectos, programas, materiales curriculares, estrategias de enseñanza y aprendizaje, modelos didácticos, otra forma de organizar y gestionar el curriculum, el centro y la dinámica del aula.

Hallazgo 6. En algunas ocasiones la innovación se impulsa desde la idea, la cual no surge de quien lleva la investigación a su cargo, sino de un tercero, un estudiante, un compañero de trabajo, por ello, es importante conocer a los estudiantes, leerlos, escucharlos, de manera que se pueda poner en práctica la capacidad para observar, motivar, orientar y ayudarlos a construir nuevas teorías procesos de investigación para mejorar casos de apatías en los estudiantes.

Hallazgo 7. Existen dimensiones claras para 
incentivar la investigación, en cuanto al entorno, referido a la comunidad local como a la sociedad en general; a las instituciones de las cuales tiene algún tipo de dependencia (supervisor, jefatura, ministerio de educación, etc.), y a otras instituciones de la comunidad con las que pueda tener relación. Hasta un cierto punto las escuelas tienen una relación informal y no vinculante con las personas y las organizaciones.

Hallazgo 8. No hay mucha claridad en la situación real acerca de las dimensiones necesarias que impulsan una investigación desde la innovación, por ello, si una escuela rompe con las expectativas, normas y tradiciones, y con la idea que tenemos de lo que una escuela debe ser, se generará una fuerte reacción.

Hallazgo 9. El dilema que se les presenta a los directivos es hasta dónde deberán "abrir" las escuelas al entorno y hasta dónde deberán "protegerlas", pues siendo una institución pública deben atender el corto y el largo plazo, como así también mantener la igualdad de oportunidades y, al mismo tiempo, procurar una alta calidad.

Hallazgo 10. Una dimensión importante son los valores, ellos están relacionados con la concepción que se tenga de la enseñanza y el aprendizaje. En este campo el desafío reside en clarificar aquellas áreas en que es necesario que los valores sean compartidos por todos, generando normas comunes. Por otro lado, comprender y aceptar diferentes actitudes y normas allí donde la comunidad educativa considera que deben quedar libradas a la voluntad de cada persona o grupo.

Hallazgo 11. Las relaciones humanas que presentan los problemas más profundos, en conflictos no resueltos en torno a los valores; en estructuras que no se adaptan a las necesidades; en las relaciones con la comunidad local o las autoridades. Es tarea de la conducción de la escuela la de encontrar caminos para crear un equilibrio entre los valores, las estructuras y las relaciones humanas.

Hallazgo 12. Desde la realidad algunos de los cambios que se pueden mencionar, son la apropiación en los estudiantes a la tecnología, que los docentes encuentran alternativas metodológicas y estratégicas para ser más dinámicos en el aula de clase, además extienden su experiencia a otros compañeros. Esto ha provocado cambios incluso a nivel social, genera personas más críticas, personas más conscientes.

Hallazgo 13. Es fundamental para el estudio del cambio y la innovación, donde consigna que aquellos que propugnan cambios estructurales (segundo orden) en las escuelas, pueden sufrir de un sentimiento de impotencia. Si bien el análisis que él hace podría sugerir un cierto pesimismo respecto de los cambios que pueden ser efectuados en las escuelas, en realidad, piensa que el cambio tal cual él lo considera debería causar optimismo respecto de lo que sí puede ser cambiado dentro de las estructuras existentes.

Hallazgo 14. Los cambios verdaderos, son aquellos que tratan de hacer más eficiente y más efectivo lo que ya existe, sin alterar sustancialmente las formas en que los adultos y los niños desempeñan sus roles, por ejemplo: contratar mejores docentes y directivos, aumentar los salarios, distribuir los recursos equitativamente, elegir mejores libros de texto, agregar o quitar contenidos y prácticas de aula, organizar las actividades y las personas de manera más eficiente, e introducir nuevas formas de evaluación y entrenamiento.

Hallazgo 15. Es importante, no rendirse y no entender que los procesos de investigación tienen resultados lentos y paulatinos porque 
se trabaja con humanos, hacer preguntas claras, delimitar, formar equipos que apacigüen la probabilidad de dilución frente al proceso de investigación. Esto es que, si se transforman las prácticas pedagógicas desde la realidad, las clases semipresenciales pasan hacer espacios alternativos que abren paso a que otro pueda indagar.

Hallazgo 16. La investigación escolar como una estrategia pedagógica conforma el último enfoque de investigación en el aula, en este docente y estudiantes indagan sobre los objetos de estudio de las diferentes áreas curriculares, bien sea acerca de los postulados teóricos trabajados en clase, aspectos de aplicación o verificación de la teoría.

Hallazgo 17. El docente puede asumir el liderazgo del proceso al asumir el rol de investigador principal, y a través de este involucrar los estudiantes para que desarrollen proyectos, también puede incentivarlos para que formulen desde sus intereses y motivaciones problemas o preguntas de investigación en relación con temáticas asociadas al currículo, en este caso el docente acompaña el proceso mediante asesorías oportunas para su desarrollo.

\section{Reflexiones finales}

Cuando se habla de la Compresión Lectora, se habla de que toda innovación implica un cambio intencional (Vogliotti y Macchiarola, 2003; Salinas, 2004; Castrillón, y Mares, 2013; Moreno, 2015; Imbernón, 1996), ya que un cambio a secas, puede darse más allá de la voluntad de los agentes. Al mismo tiempo el cambio que origina una innovación reviste un carácter positivo, de mejor, es decir, no se realiza una innovación con el fin de empeorar una situación.

Por tanto, el cambio no implica necesariamente una mejora, la mayoría de las personas ven al cambio como algo positivo, sin embargo, Martínez (2010) considera que "el cambio puede ser o no una mejora" (p. 11), por lo tanto, reside en la mente de quien lo realiza. La innovación en instituciones educativas, tiene una íntima relación con el sector tecnológico, aun cuando los objetivos suelen ser difusos y estar en mutuo conflicto; los objetivos de corto plazo no están necesariamente de acuerdo con los de largo, por esto es difícil descubrir los efectos no deseados de las innovaciones educativas.

Indudablemente, es la inducción hacia cambios funcionales novedosos, (Medina y Espinosa, 1994) es el volver a trabajar sobre campos de acción conocidos para aplicarlos a nuevas circunstancias, y la creación de nuevas formas de percibir y acercarse a los problemas. En parte, la innovación puede ser explicada como el aprovechamiento creativo de oportunidades y posibilidades, de tal forma que favorecen la improvisación.

En mayor medida, la innovación es el resultado de acciones preestablecidas; se orienta hacia una adaptación flexible, hacia la experimentación, y el cambio guiado. (García, 2001). Ahora bien, la innovación es como una serie de intervenciones, decisiones y procesos, con cierto grado de intencionalidad y sistematización, que tratan de modificar actitudes, culturas, contenidos, ideas, modelos y prácticas pedagógicas,

A su vez, de introducir en una línea renovadora, nuevos proyectos, programas, materiales curriculares, estrategias de enseñanza y aprendizaje, modelos didácticos, otra forma de organizar y gestionar el curriculum, el centroy la dinámica del aula. Existen dimensiones que impulsan la innovación en los procesos de enseñanza-aprendizaje, estos son, el entorno, los valores, la estructura, las relacionesy las estrategias, con estas se pueden generar los cambios necesarios que permitan el avance en los procesos educativos (Ambriz, 2009). 
Estos cambios es un primer orden, son aquellos que tratan de hacer más eficiente y más efectivo lo que ya existe, sin alterar sustancialmente las formas en que los adultos y los niños desempeñan sus roles, por ejemplo: contratar mejores docentes y directivos, aumentar los salarios, distribuir los recursos equitativamente, elegir mejores libros de texto, agregar o quitar contenidos y prácticas de aula, organizar las actividades y las personas de manera más eficiente, e introducir nuevas formas de evaluación y entrenamiento.

Los cambios en segundo orden, se buscan modificar la forma fundamental en la que se relacionan las organizaciones. Reflejan una insatisfacción con las configuraciones existentes; introducen nuevos objetivos, estructuras y roles, que transforman las maneras conocidas de hacer las cosas hacia nuevas formas de resolver los problemas persistentes.

Por otro lado, las estrategias de compresión lectora son procedimientos usados de forma premeditada y flexible por parte los educadores, pueden también ser utilizadas antes del momento de la enseñanza, con el objeto de impulsar el conocimiento previo, o también durante la enseñanza para asistir la atención o el manejo de la información, o al final de la enseñanza para fortificar el aprendizaje de la información nueva.

En este sentido, los docentes han de estar en capacidad de alentar la investigación, la participación y la innovación en el alumnado. Todo este proceso se genera con la finalidad de ir de un modelo tradicional de educación que ubica al docente como único responsable del trasmitir conocimiento dentro el aula, a un modelo alternativo que facilite al estudiante hacer uso del método científico con el propósito de que aprenda a investigar investigando.

Por otro lado, hay un gran número de estrate- gias de enseñanza; en especifico tres formas posibles de desarrollar la investigación de aula; la primera la investigación del docente sobre su práctica, la segunda la investigación del docente sobre las prácticas de los estudiantes, y por último la investigación en la que el docente acompaña procesos investigativos de los estudiantes.

Finalmente, el potencial humano se devela desde que la investigación científica dejo de ser una labor aislada y se convirtió en una profesión socialmente organizada comprometida con la búsqueda del saber verdadero y encaminado a la producción y socialización de sus hallazgos sobre las diferentes líneas de interés investigativo.

\section{Recomendaciones}

Luego de plasmar las reflexiones finales del estudio, donde se conoció la innovación de enseñanzas y aprendizajes en las prácticas investigativas de docentes de la Ciudad de Medellín, se recomienda tanto a la institución que se tomó como objeto de estudio, como a otras instituciones educativas, que deseen sumarse al mejoramiento de la formación de sus estudiantes como lectores autónomos, las siguientes recomendaciones:

- Incentivar permanentemente la innovación a través de diferentes tipos de cambios para acompañar el proceso, garantizando así el desarrollo de sus capacidades mediante el uso de diversas estrategias de enseñanza - aprendizaje.

- Comprender como los profesores pueden mejorar la innovación educativa, utilizando las estrategias idóneas y asertivas para desarrollarla, dedicándole el tiempo y el apoyo que se requiera.

- Promover cambios necesarios, no solo en las asignaturas que se estén cursando, sino los di- 
versos programas, acciones, planes y proyectos institucionales, para fortalecer el proceso de enseñanza-aprendizaje.

- Crear climas adecuados para trabajar de forma respetuosa y cercana, permitiendo la innovación y participación de todos por igual, generando espacios de aprendizajes mediante grupos de distintos niveles de compresión.

- Evaluar constantemente las debilidades, amenazas, aciertos, limitaciones, avances, oportunidades, desventajas, para poder corregir y ayudar en las áreas donde se necesite, reconociendo que la innovación parte de un proceso de revisión y monitoreo continuo del entorno, lo que permitirá avanzar con la dinámica educativa.

- Identificar las áreas con mayor dificultad para desarrollar proyectos innovadores, de manera que se puedan preparar actividades especiales para atender dicha debilidad.

- Utilizar diversas dimensiones de la innovación para ir mejorando las estrategias de enseñanza-aprendizaje que cuenten con una menor capacidad en relación este propósito.

- Incentivar a los docentes, estudiantes, así como a todos los involucrados del hecho educativo a tener una mayor capacidad de adaptación a los cambios innovadores, al mismo tiempo que ayuden a los que presenten dificultad, sin olvidar que la guía principal la debe tener el docente dentro del aula y los gerentes a niveles institucionales.

- Tener claro qué se quiere obtener con la innovación que se realiza, en especial con los cambios de fondo que se envían a realizar y las que se realizan en el aula.

- Proponer situaciones reales para fortalecer los cambios innovadores, trayendo a colación experiencias vividas por cada participante y analizándolas para mejorar los puntos críticos.

- Propiciar que los docentes actúen de forma independiente y autónoma en cualquier situación de aprendizaje.

\section{Teoría sustantiva de la innovación en los procesos de enseñanza-aprendizaje en las prácticas investigativas de los docentes}

El poder que representa para un individuo la capacidad de innovar, es lo que le permite expandir y enriquecer los modelos del mundo y su perspectiva con respecto a las diferentes oportunidades que se le presenten. La innovación educativa pretende mudar o alterar algo, introduciendo novedades, específicamente en el capo educativo la innovación está al servicio de las estrategias prácticas que requieren de un enfoque conciso para una propuesta que plantea un cambio de paradigma.

En este sentido, es así como en el campo educativo surgen modelos que permiten crear un impacto positivo que aporte conceptos significativos a los procesos de enseñanza-aprendizaje en las practicas investigativas de los docentes que tenga como proceso fundamental la innovación, es decir, que a través de este paradigma se tenga la oportunidad de crecer continuamente, en los aspectos emocionales, psicológicos, intelectuales, profesionales y al mismo tiempo contribuyen al progreso en la calidad intelectual y educativa del talento humano de las instituciones educativas.

Aunado a esto, hace referencia a tres usos relacionados entre sí; la innovación en relación a una invención, es decir, al proceso creativo por el cual dos o más conceptos existentes o entidades son combinados en una forma novedosa, para producir una configuración desconocida previamente. En segundo lugar, la innovación es descrita como el proceso por el cual una innovación existente llega a ser parte 
del estado cognitivo de un usuario y de su repertorio conductual.

Y, por último, una innovación es una idea, una práctica o un artefacto material que ha sido inventado o que es contemplado como novedad, independientemente de su adopción o no adopción. De este modo, el concepto de innovación aparecerá relacionado a estos tres usos: la creación de algo desconocido, la percepción de lo creado como algo nuevo y la asimilación de ese algo como novedoso.

Lo anterior permite explicar entonces, que la innovación educativa aparece mucho más ligada a los dos últimos usos, por ejemplo, a la implementación del trabajo grupal como estrategia de enseñanza-aprendizaje para incentivar las prácticas de investigación docente. Cualquier innovación educativa que aporte novedades que provocan cambios; representa un avance en la gestión del conocimiento, esos cambios pueden ser drásticos, en cuanto a que se deja de hacer las cosas como se hacían antes para hacerlas de otra forma o progresivos ya que, se hacen de forma parecida, pero introduciendo alguna novedad; en cualquier caso, el cambio siempre mejora lo cambiado; es decir, la innovación sirve para mejorar algo.

Tal como se señala, una forma de incorporar novedades que produzcan cambios progresivos consiste en incorporar prácticas investigativas que han demostrado éxito en otros campos; en este sentido utilizar las dimensiones del cambio que se consideren innovación, ya que mejoran el servicio. De lo anterior se desprende, que llevando todo esto al proceso educativo y descartando que en un corto espacio de tiempo se produzca un cambio drástico; la innovación educativa introduciría novedades que mejorarían el proceso formativo; pero siempre con un coste asequible; la de prácticas de investigación podría producir esa innovación educativa.

Contextualizando, desde el punto de vista social-educativo, el éxito de un proceso formativo para un estudiante consiste en la aprobación y promoción de su curso escolar, así como, se convierte igualmente en el propósito de sus padres y educadores; por lo que la innovación educativa pretende ir más allá, persigue un cambio en la forma de innovar en las estrategias de enseñanza-aprendizaje.

En este sentido, las prácticas de investigación involucran dos aspectos relevantes, por una parte, la idea de investigar de algún modo, la organización, la planificación, la dirección y el control de procesos para conformar o disponer de ciertos objetivos. Por otro lado, se pone de manifiesto que una institución, como cualquier ser humano, está sometida a una dinámica en la que del exterior y del interior mismo, capta o percibe información, la reconoce, la organiza, la almacena, la analiza, la evalúa y emite una respuesta al exterior, procurando un resultado.

Actualmente, interesa sobre todo el aprendizaje basado en innovación, no en vano una de las tendencias más sólidas durante la última década en las instituciones educativas ha sido el de los programas de formación permanente del personal y demás postulados relativos al aprendizaje en equipo. En el ámbito educativo se ha valorado el recurso humano como algo dinámico, sin embargo, se valora más la capacidad del aprendizaje innovador que los conocimientos adquiridos. Es más importante innovar para gestionar el aprendizaje, ya que esto significa conocer el conocimiento, más que poseer una colección de conocimientos.

De acuerdo con esto, la innovación en las estrategias de enseñanza aprendizaje para mejorar los procesos de investigación en las prácticas docentes necesita de la aplicación del 
conocimiento existente en la obtención de resultados y la innovación sistémica o la definición de los nuevos conocimientos necesarios, su factibilidad y del método para hacerlo eficaz. Adicionalmente se puede agregar, que la tarea de innovar es una tarea de saber desarrollar nuevos y diferentes procesos y productos dentro de las organizaciones.

En particular, se debe analizar la información que se maneja en la institución, ya que, el éxito de esta resulta de su capacidad de identificar y respetar los individuos y de la capacidad de integrarlos en un todo. Innovación, en un sentido general, supone que una organización se dote de tres funcionalidades estratégicas distintas, reunidas en una sola, esto es, la reutilización o realimentación del valor añadido que la institución genera y adquiere y que representa el capital intelectual de la misma, al servicio de la resolución de nuevos problemas, incrementando de esta manera el valor añadido de los servicios producidos y el rendimiento de dicha actividad.

Por otra parte, la investigación, que en la institución son los productores de valor añadido y/o son responsables de tomar decisiones críticas, sobre la base de una adecuada disposición de información diversa y una rápida respuesta. Por último, el acceso unificado a todas y cada una de las capas de información tejidas sobre la estructura organizacional.

En este sentido, en la práctica, el individuo en la institución educativa se enfrenta a todo el conocimiento o a una parcela de él, por eso la innovación educativa en pro de la investigación debe ser también un instrumento de estudio y avance. El soporte de un sistema lo constituye la información documental que a diario es generada en las escuelas, hecho que lamentablemente muy poco se cumple dentro de la realidad educativa. De manera que sí la misma se maneja en forma idónea se podrá mantener más accesible y más segura, por lo tanto, más viva.

En resumen, un sistema de gestión del conocimiento basado en procesos de innovación permite la reutilización de la información almacenada en la institución educativa y su incorporación en los procesos funcionales y operacionales integrando los sistemas de información existentes y permitiendo la durabilidad de la información y el conocimiento, así mismo entendiendo que el aprendizaje está en el manejo adecuado de la información y que esta genere aportes, así como cambios efectivos.

Desde todo punto de vista, la innovación refiere a aquel cambio que introduce alguna novedad o varias, cuando alguien innova aplica nuevas ideas, productos, conceptos, servicios y prácticas a una determinada cuestión, actividad o negocio, con la intención de ser útiles para el incremento de la productividad. Una condición esencial de la innovación es su aplicación exitosa a un nivel comercial, sin embargo, en el campo educativo, realmente es importante su aplicación, para ello se necesitan de factores que permitan su aplicación cónsona con la realidad educativa.

Para, innovar con éxito es importante saber, el que empieza debe tener claro que trabajará sobre tiempo si quiere lograr el objetivo propuesto; ganas de salir adelante; motivación por ser independiente, capacitación continua del empresario y de los trabajadores; utilizar los conocimientos aprendidos como empleado de otra organización a la hora de crear la propia empresa, lo anterior presume que si estos factores suceden es porque los innovadores profesionales arrancan con una ventaja competitiva.

De igual manera, es necesario buscar nuevas oportunidades para innovar en lo que son 
necesidades básicas, considerando que las alianzas estratégicas son una buena alternativa. También es necesario para lograr la meta trazada, utilizar estrategias didácticas y pedagógicas que consoliden el proceso. Por ello, el éxito en los innovadores depende del conocimiento y análisis por parte del innovador; la creatividad para mezclar y combinar de manera especial, diferenciante y preferible dichas variables; la capacidad para ejecutar, o saber hacer que ejecuten la combinación adecuada.

Por todo ello, su aporte radica en combinar con creatividad lo que ya existe y el resultado final parece ser algo superior, nuevo, mejor y diferente que el producido por otra persona que utiliza los mismos elementos pero que los combina, de diferente forma. Entre los elementos básicos de un innovador, el autor destaca la generación de la idea, la búsqueda de ventajas competitivas, el posicionamiento, la política de recursos humanos, el servicio que se ofrece, los atributos principales de preferencia, las técnicas de enseñanza, el análisis de los conocimientos y la aplicación de proyectos.

En correspondencia, existen factores claves de éxito para quien implementa una innovación educativa, como que son muy trabajadores, tienen mucha energía puesta en el trabajo. En segundo lugar, destaca que son personas muy dinámicas, y finalmente, en tercer lugar, son bastante agresivos, cuando son atacados, rápidamente responden, no se quejan, actúan.

En definitiva, según los autores analizados entre los factores claves de éxito de innovación se destacan especialmente características personales, de información y conocimiento de adaptación al cambio y actualización tecnológica. Puntualizando, a efecto de esta investigación los factores claves para la innovación como proceso de la gestión de cono- cimiento que a juicio de este autor se deben considerar son: la competitividad, dirección, organización, competencias; los mismos serán desarrollados a continuación.

Desde otro punto de vista, la innovación se ha convertido en un asunto de primer orden para las instituciones educativas que cada día se ven sometidas a la creciente globalización y la aparición de nuevos competidores, los cuales no desestiman esfuerzos por mejorar continuamente. También las naciones han entrado en la onda competitiva e incluso se ha diseñado un ranking mundial de competitividad en pro de la innovación.

El uso del término innovación educativa, consiste en la capacidad de las instituciones educativas, en este caso particular, para alcanzar un éxito sostenido contra (o en comparación con) sus competidores foráneos sin afectar su calidad educativa, con la posibilidad que tienen sus estudiantes para alcanzar un nivel de vida elevado y creciente.

Pero la innovación no se limita al mero hecho de competir, sino que va mucho más allá debido a que implica un proceso de mejora continua, de comparación de resultados y de búsqueda permanente de la excelencia. La innovación inicia por el individuo que se esfuerza todos los días por ser el mejor de la clase y tener prospectiva de superación personal y profesional.

El divorcio entre la educación y el sistema productivo cada vez se ha hecho más profundo. La agenda educativa del país parece darle la espalda a la necesidad de convertir el sistema de enseñanza en una palanca de desarrollo que genere una cultura de la productividad y la eficiencia. Si la nación no es capaz de entender la necesidad de reestructurar el sistema educativo en función de vincular el esfuerzo individual, la eficiencia y la productividad. 
Tal como se viene señalando, la innovación educativa; es una fórmula de cómo la empresa va a competir para alcanzar sus objetivos. Para que el gerente educativo elabore sus objetivo o meta, es necesario que conozca el desempeño de su personal, definiendo el desempeño, como aquellas acciones o comportamientos de los docentes dentro de sus cargos que ocupan en la institución, pero para conocer el desempeño docente, es necesario que existan una serie de competencias que ayuden a conocer esas acciones que se evalúan en un desempeño del docente.

Por ello es necesario, conocer el significado de la innovación ejercida por el docente; la cual son actuaciones integrales que permiten identificar, interpretar, argumentar y resolver problemas del contexto como idoneidad y ética, integrando los momentos contextuales como son el saber ser, que es el modo de pensar y sentir, el saber hacer; las habilidades y destrezas del docente, el saber conocer, son las características conductuales, cognitivas, motoras y modales.

Cuando se analiza que, dentro de la innovación educativa del docente en sus prácticas de investigación, se evalúa de diferentes maneras, tomando en cuenta sus características, habilidades y destrezas. Todas estas competencias que poseen los docentes deben evaluarse imparcialmente con justicia y honestidad, entendiendo la evaluación, como un proceso continuo, mediante el cual se describen las habilidades destrezas de los docentes, para lograr los objetivos propuestos.

Entonces, según las premisas anteriores para que el docente evalúe el desempeño de un estudiante, debe tener una misión, visión que permita aplicar un plan estratégico competitivo, basado en las competencias a evaluar. Dentro de este plan estratégico, el Docentes, debe tener claro lo que es la innovación, para fijar los objetivos, los avances del proceso de evaluación y la fecha de la realimentación.

En definitiva, la innovación educativa como factor clave de progreso en los procesos de investigación requiere un equipo directivo dinámico, actualizado, abierto al cambio organizativo y tecnológico, y consciente de la necesidad de considerar a los miembros de la organización como un recurso de primer orden al que hay que cuidar. Sin embargo, se puede afirmar que este suele ser uno de los puntos débiles de un elevado número de instituciones que ha desaparecido o tienen problemas de supervivencia.

En resumen, el docente cuando innova, planifica y toma en cuenta los miembros de la organización, posee la habilidad de tener una visión clara, que le permita llevar a cabo la construcción de una visión compartida, es decir, la ejecución de proyectos o planes. Por lo mismo, es necesario que estos, promuevan dentro de las escuelas esta visión compartida, que no es más que lo que se quiere lograr o a dónde se quiere llegar, implementando para ello Proyectos de investigación, basados en una realidad educativa y comunitaria. 


\section{Referencias}

Ambriz, G. S. (2009). El desarrollo organizacional: una estrategia de cambio para las instituciones documentales. In Anales de documentación (Vol. 12, pp. 235-254).

Arnold, M. (1997). Introducción a las epistemologías sistémico/constructivistas. Cinta de Moebio. Revista de Epistemología de Ciencias Sociales, (2).

Arráez, M., Calles, J., \& Moreno de Tovar, L. (2006). La Hermenéutica: una actividad interpretativa. Sapiens, 7(2), 177-181.

Bonilla, Castro y Rodríguez (2005). Más allá del dilema de los métodos: la investigación en ciencias sociales. Tercera Edición ampliada y revisada. Universidad de los Andes. Editorial Grupo Norma. Bogotá, Colombia.

Castrillón, M. A. G., \& Mares, A. I. (2013). Innovación empresarial, difusión, definiciones y tipología: una revisión de literatura. Dimensión empresarial, 11(1), 45-60.

Colciencias. Programa Ondas. (2016). Niños, niñas y jóvenes que investigan: lineamientos pedagógicos del programa ondas. Editorial Edeco Ltda. 37-50. Recuperado de http://repositorio.colciencias.gov. co:8081/jspui/handle/11146/368

Cordero, I. (2013). Didáctica y enseñanza del Derecho en las universidades. Pensamiento Americano. 6(11) 29-34

De la Fuente Arias, J., Martínez, M. M. V., \& Cardelle-Elawar, M. (2018). Aportaciones de la Psicología de la Innovación y del Emprendimiento a la Educación, en la Sociedad del Conocimiento. Electronic Journal of Research in Education Psychology, 10(28).

Freinet, C. (1975, 1977). Técnicas Freinet de la escuela moderna. Siglo Veintiuno Editores. Primera Edición. México.

García Fallas, J. (2001). Supuestos epistemológicos que subyacen a la innovación educativa. Revista Electrónica" Actualidades Investigativas en Educación", 1(1).

Higuera Ojito, V. (2013). Análisis económico de la demanda educativa en programas de Ciencias Económicas: un modelo de elección basado en un logit binomial. AdGnosis, 2(2), 13-21

Imbernón, F. (1996). En busca del discurso educativo: la escuela, la innovación educativa, el currículum, el maestroy su formación.

Krause, M. (1995). La investigación cualitativa: un campo de posibilidades y desafíos. Revista Temas de educación, 7(7), 19-40.

Martínez, E. D. (2010). " Innovación Educativa a Través de la Implementación de Modelo Educativo por Competencias en el TEST: un Caso de éxito o Fracaso"Edición Única.

Martínez, M. (1999). Paradigmas y Métodos de Investigación en Tiempos de Cambios. Editorial CEC SA. Venezuela.

Medina Salgado, C., \& Espinosa Espíndola, M. T. (1994). La innovación en las organizaciones modernas.

Mora, J. G. (2004). La necesidad del cambio educativo para la sociedad del conocimiento. Revista Iberoamericana de educación, 35(2), 13-37.

Moreno, M. G. (2015). Formación de docentes para la innovación educativa. Sinéctica, (17).

Programa Ondas. (2009). La ciencia, la tecnología y la innovación en las culturas infantiles y juveniles de Colombia. Evaluación de Impacto del Programa Ondas. Recuperado de http://repositorio.colciencias. gov.co:8081/jspui/bitstream/11146/347/1/253-2\%20\%20 KP\%20plegable.pdf

Rodríguez, G., Gil, J., \& García, E. (1999). Proceso y fases de la investigación cualitativa. Metodología de la investigación cualitativa. Archidona: Aljibe, 149-166.

Rodríguez, M. B.y Ungaro A. M. (2010). Accesoy permanencia en una educación de calidad. Congreso lberoamericano de Educación.

Salinas, J. (2004). Innovación docente y uso de las TIC en la enseñanza universitaria. International Journal of Educational Technology in Higher Education (ETHE), 1(1).

Strauss, A. y Corbin, J. (2002). Bases de la investigación cualitativa: técnicas y procedimientos para desarrollar la teoría fundamentada. Medellín: Universidad de Antioquia.

Vogliotti, A., \& Macchiarola, V. (2003). Teorías implícitas, innovación educativa y formación profesional de docentes. In Ponencia Congreso Latinoamericano de Educación Superior. Argentina: Universidad de San Luis.

2018, Vol. 11(21) 153-168 @ The Author(s) 2018 Reprints and permission: www.americana.edu.co

https://www.coruniamericana.edu.co/publicaciones/ojs/index.php/pensamientoamericano/index 